\title{
Gestión de Inundación por Caudales Máximos en la Subcuenca Baja de la Quebrada del Río Huaycoloro - Río Rímac
}

\author{
Flood Management for Maximum Flows in the Lower Subbasin River Gorge Huaycoloro - Rimác \\ River
}

\author{
Gina Quincho
}

Universidad Nacional Federico Villarreal, Lima, Perú

DOI: https://doi.org/10.33017/RevECIPeru2014.0014/

\section{Resumen}

El objetivo de la presente tesis es investigar la gestión de inundación por caudales máximos en la subcuenca baja de la quebrada del río Huaycoloro, con la finalidad de contribuir a mejorar la calidad de vida de los centros poblados como Cajamarquilla, Jicamarca y Huachipa; está investigación se ha estructurado en 4 capítulos. El primer capítulo desarrolla la problemática, los objetivos, la hipótesis que se muestran con sus respectivas variables y se justifica su relevancia y la importancia de esta investigación. En el segundo capítulo se muestra el marco conceptual y el marco normativo ambiental que sustenta los criterios y toma de decisiones para las propuestas de conservación y defensas rivereñas. En el tercer capítulo se describe la metodología utilizada por cada objetivo planteado, asimismo se detalla el tipo de investigación y métodos, los materiales y equipos; etapas en la realización del presente estudio. El cuarto capítulo, describe el diagnóstico ambiental de la Subcuenca Huaycoloro, que tiene un área $492.9 \mathrm{~km} 2$ y la longitud del río principal es de $49.7 \mathrm{~km}$, las características morfológicas le dan un área mayor en porcentaje de 54 \% (colina y montaña - vertiente montañosa empinada a escarpada); se presentan 3 climas de un semiseco a un árido deficiente de lluvias; en su geología presenta predominancia de rocas ígneas; así mismo en las características socio económicas resalta que 38,436 habitantes que equivale al $60 \%$ de pobreza extrema; la precipitación máxima en 24 horas de la subcuenca varia de $5 \mathrm{~mm}$ hasta $30 \mathrm{~mm}$, se estimó el caudal máximo dando como resultado para un periodo de retorno, para 10 años de $75 \mathrm{~m} 3 / \mathrm{s}$, para 50 años 104 y finalmente para 100 años $120 \mathrm{m3} / \mathrm{s}$. Asimismo, se establece los criterios para la gestión de riesgos en la cual comprende el análisis peligros y vulnerabilidad, identificando el riesgo en zonas inundables que para 50 años le corresponden 37 ha de inundación en el tramo crítico. Finalmente se desarrollan los tipos de propuestas de conservación en suelos inundados y erosionados como medidas estructurales (gaviones, llantas), y no estructurales (agroforestería) con la finalidad de mitigar daños por desborde en la Subcuenca.

Descriptores: Gestión, inundación, peligro, vulnerabilidad, riesgo, gaviones, agroforestería.

\section{Abstract}

The objective of this thesis is to investigate the management of flood peak flows in the lower subbasin Gulch Huaycoloro river, in order to help improve the quality of life of the towns as Cajamarquilla Jicamarca and Huachipa, this research is structured in 4 chapters. The first chapter develops the problem, objectives; hypotheses are shown with their respective variables and justify their relevance and importance of this research. In the second chapter the conceptual framework and the environmental regulatory framework that supports the decision-making criteria and proposals for conservation and riverine defenses shown. In the third chapter the methodology used for each objective proposed is described, also the type of research and methods, materials 
and equipment is detailed, steps in conducting this study. The fourth chapter describes the environmental diagnosis of Huaycoloro subwatershed, which has a $492.9 \mathrm{~km} 2$ area and length of the main river is $49.7 \mathrm{~km}$, the morphological features give you a greater area percentage of $54 \%$ (hill and mountain - Mountain slope steep to steep) 3 climates presents a semi- arid to poor rains, in geology presents predominance of igneous rocks, likewise in the socio economic characteristics highlights that 38,436 inhabitants equivalent to $60 \%$ of extreme poverty; precipitation maximum within 24 hours of various sub from $5 \mathrm{~mm}$ to $30 \mathrm{~mm}$, the maximum flow resulting to a return period to 10 years of $75 \mathrm{~m} 3 / \mathrm{s}$, for 50 years and eventually 104 to 100 years $120 \mathrm{~m} 3$ / estimated s. Also, the criteria for risk management which includes the hazards and vulnerability analysis, identifying risk in flood zones that correspond to 50 years has 37 critical flood stage is set.

Keywords: Management, flood hazard, vulnerability, risk, gabions, agroforestry.

\section{Introducción}

Las inundaciones están a lo largo del territorio peruano debido a sus características geológicas, geomorfológicas, climáticas, etc. Que condicionen un comportamiento muy activo representada por inundaciones.

La inadecuada interrelación del hombre con la naturaleza y su desconocimiento sobre aspectos básicos de seguridad física ponen en evidencia la vulnerabilidad de los centros poblados ante la ocurrencia de desastres que en muchas ocasiones alcanzan niveles catastróficos en países donde no existe la cultura de educación ambiental y prevención por inundación.

La cuenca del Río Rímac donde se ubica el área de estudio, se dan prolongados periodos de estiaje con periodos de avenidas que afectan las áreas adyacentes, por esta razón el objetivo de la tesis es investigar la gestión de inundación por caudales máximos en la Subcuenca baja de la quebrada del río Huaycoloro con la finalidad de mitigar los riesgos y contribuir a mejorar la calidad de vida de la población, debido a que la Subcuenca Huaycoloro es muy dinámica, y con diversos fenómenos de remoción en masa que en las últimas décadas ha causado huaycos e inundaciones, causando grandes pérdidas económicas, especialmente a los centro poblados de Cajamarquilla y Huachipa; Así como también perjudicando a los distritos cercanos como Campoy y Zárate. Otros investigadores como O, Vivar 1975; O, Vera 1989; A, Ricalde 1993; W, Gómez 2000; D, Garcia2002; N, Zamora y otros realizaron una serie de investigaciones específicamente relacionados a la cuenca del río Rímac y algunas quebradas investigando estas áreas sin tomar en cuenta la zona en estudio.
En la presente tesis revela la importancia por la problemática permanentemente a inundaciones debido al caudal máximo y arrojo de basura al cauce del río y la poca protección del cauce. En este sentido se ha utilizado la información existente y similar de acuerdo con los objetivos planteados, manejando los aspectos de cobertura vegetal, pendiente, precipitación y geomorfología, hidráulicos, forestales y socioeconómicos con la cartografía digital correspondientes que permitan generar modelos matemáticos que definan espacialmente zonas de peligros inundables, así como áreas de vulnerabilidad y riesgo; también se planteó propuestas de medidas para mitigar desastres ocurridos por inundación en el área de investigación.

\section{Diagnóstico Ambiental}

Se describe el procedimiento experimental y el equipamiento utilizado. Aquí no se muestran los resultados sino se describe la metodología empleada en el trabajo experimental.

\subsection{Ubicación geográfica y política.}

Geográficamente la Subcuenca Huaycoloro, pertenece a la cuenca baja del río Rímac, y está caracterizada por una morfología que varían de colinoso y ondulado a laderas inclinadas $y$ accidentadas, con superficies de erosión desarrolladas, tiene sus orígenes en las inmediaciones de las quebradas Batán, Tranca, Michura. Colca, Asma, entre otros, sobre los 3500 m.s.n.m. de altitud.

Limita por el norte y oeste, con la cuenca del río Chillón; por el este con la subcuenca del río Santa Eulalia y, por el sur, con el área de la propia cuenca baja del río Rímac. 
Políticamente se encuentra ubicada en los centros poblados de Cajamarquilla, Jicamarca y Santa María de Huachipa, entre los distritos de Lurigancho Chosica y San Antonio.

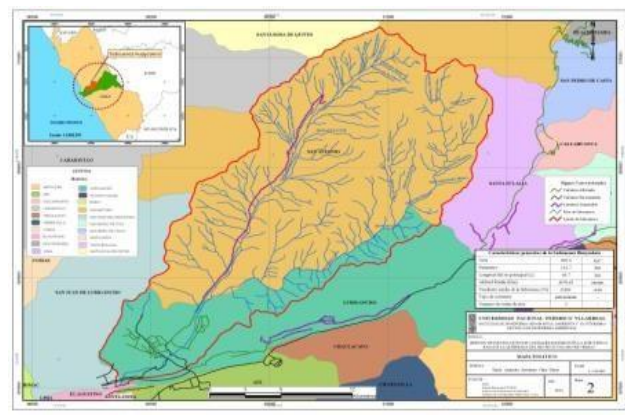

\subsection{Parámetros geomorfológicos}

La morfología de la subcuenca Huaycoloro Jicamarca se forma por la confluencia de la microcuenca Huaycoloro y microcuenca río seco definida por su forma, mediante el cálculo de sus parámetros geomorfológicos, con los cuales será posible determinar el contexto físico del área de estudio como el escurrimiento y la velocidad de respuesta, es decir, la respuesta hidrológica.

Tabla 1: Resumen de parámetros geomorfológicos de la subcuenca Huaycoloro que identifican la morfología y la red hídrica. Fuente: Elaboración propia.

\begin{tabular}{|c|c|c|c|c|}
\hline № & \multicolumn{2}{|c|}{ Parámetros } & Resultados & Unidades \\
\hline 1 & \multicolumn{2}{|l|}{ Área } & 492.9 & $\mathrm{~km}^{2}$ \\
\hline 2 & \multicolumn{2}{|l|}{ Perímetro } & 112.7 & $\mathrm{~km}$ \\
\hline 3 & \multicolumn{2}{|c|}{ Longitud del río principal (L) } & 49.7 & $\mathrm{~km}$ \\
\hline 4 & \multicolumn{2}{|c|}{ Ancho promedio de la cuenca (Ap) } & 9.9 & $\mathrm{~km}$ \\
\hline 5 & \multicolumn{2}{|c|}{ Coeficiente de Compacidad (Kc) } & 1,4 & - \\
\hline 6 & \multicolumn{2}{|l|}{ Factor de Forma (Ff) } & 0,20 & - \\
\hline 7 & \multicolumn{2}{|l|}{ Grado de Ramificación } & 80,5 & $\mathrm{~km}$ \\
\hline \multirow[t]{2}{*}{8} & \multirow[t]{2}{*}{ Rectángulo Equivalente } & Lado mayor & 45.5 & $\mathrm{~km}$ \\
\hline & & Lado menor & 10.8 & $\mathrm{~km}$ \\
\hline 9 & \multicolumn{2}{|l|}{ Radio de circularidad (Rc) } & 0.49 & - \\
\hline 10 & \multicolumn{2}{|l|}{ Altitud Media $(\mathrm{Hm})$} & 1678.43 & msnm \\
\hline 11 & \multicolumn{2}{|c|}{ Pendiente media de la Subcuenca } & 0.091 & $\mathrm{~m} / \mathrm{m}$ \\
\hline 12 & \multicolumn{2}{|l|}{ Índice de pendiente (Ip) } & 0.090 & - \\
\hline 13 & \multicolumn{2}{|c|}{ Coeficiente de Masividad $(\mathrm{Cm})$} & 0.0019 & - \\
\hline 14 & \multicolumn{2}{|c|}{ Coeficiente orográfico (Co) } & 0.002 & - \\
\hline 15 & \multicolumn{2}{|c|}{ Coeficiente de Torrencialidad (Ct) } & 0.42 & - \\
\hline 16 & \multicolumn{2}{|l|}{ Tipo de corriente } & intermitente & - \\
\hline 17 & \multicolumn{2}{|l|}{ Numero de orden de ríos } & 5 & - \\
\hline 18 & \multicolumn{2}{|c|}{ Frecuencia de densidad de Ríos (Fr) } & 0.82 & Ríos $/ \mathrm{km}^{2}$ \\
\hline
\end{tabular}

\begin{tabular}{|c|l|c|c|}
\hline 19 & Densidad de Drenaje (Dd) & 1.08 & $\mathrm{~km} / \mathrm{km}^{2}$ \\
\hline 20 & $\begin{array}{l}\text { Extensión media de Escurrimiento } \\
\text { Superficial ( E ) }\end{array}$ & 0.93 & $\mathrm{Km}^{2} / \mathrm{km}$ \\
\hline 21 & Pendiente media del río principal (Sm) & 8.3 & $\%$ \\
\hline 22 & Altura media del río principal (Hmed) & 2337.5 & $\mathrm{msnm}$ \\
\hline
\end{tabular}

\section{Evaluación Hidrometeorológica}

En la subcuenca Huaycoloro no existen estaciones pluviométricas, lo cual limita el análisis de precipitaciones que permitirá conocer el patrón de este comportamiento, razón por la cual se utilizó información de estaciones pluviométricas que están dentro y fuera de la subcuenca, lo cual permiten definir la pluviometría del área de estudio en base al análisis de la altitud y latitud, y que fueron analizadas, teniendo en cuenta el periodo, la cercanía y la similitud pluviométrica.

\subsection{Determinación del caudal máximo}

Habiendo determinando cada uno de los elementos que nos permiten determinar el caudal máximo y aplicando la ecuación Racional:

Qmáx $=($ C.I.A.K $) / 3.6$

Dónde: $\mathrm{Q}$ máx: Caudal en $\mathrm{m} 3 / \mathrm{s}$; C: Coeficiente de Escorrentía; I: Intensidades (mm/hora); A: Área de drenaje $(\mathrm{Km} 2)$.

Utilizando la información de los valores $\mathrm{Ce}, \mathrm{K}$ y las áreas de las isócronas encontramos los caudales de avenida, máximo y de agotamiento, que corresponden a cada Tiempo de Retorno.

Tabla 2: Caudales máximos de diferentes tiempos de retorno. Fuente: Elaboración propia.

\begin{tabular}{|c|c|c|c|c|c|c|c|}
\hline & $\begin{array}{c}5 \text { TR } \\
\text { AÑOS }\end{array}$ & $\begin{array}{c}10 \text { TR } \\
\text { AÑOS }\end{array}$ & $\begin{array}{c}15 \text { TR } \\
\text { AÑOS }\end{array}$ & $\begin{array}{c}20 \text { TR } \\
\text { AÑOS }\end{array}$ & $\begin{array}{c}25 \text { TR } \\
\text { AÑOS }\end{array}$ & $\begin{array}{c}50 \text { TR } \\
\text { AÑOS }\end{array}$ & $\begin{array}{c}100 \text { TR } \\
\text { AÑOS }\end{array}$ \\
\hline $\begin{array}{c}\text { T } \\
\text { c }\end{array}$ & $\begin{array}{c}\text { Caudal } \\
\mathrm{m} 3 / \mathrm{s}\end{array}$ & $\begin{array}{c}\text { Caudal } \\
\mathrm{m} 3 / \mathrm{s}\end{array}$ & $\begin{array}{c}\text { Caudal } \\
\mathrm{m} 3 / \mathrm{s}\end{array}$ & $\begin{array}{c}\text { Caudal } \\
\mathrm{m} 3 / \mathrm{s}\end{array}$ & $\begin{array}{c}\text { Caudal } \\
\mathrm{m} 3 / \mathrm{s}\end{array}$ & $\begin{array}{c}\text { Caudal } \\
\mathrm{m} 3 / \mathrm{s}\end{array}$ & $\begin{array}{c}\text { Caudal } \\
\mathrm{m} 3 / \mathrm{s}\end{array}$ \\
\hline 1 & 25,5 & 37,0 & 37,8 & 42,7 & 46,0 & 51,0 & 58,4 \\
\hline 2 & 36,9 & 52,8 & 54,3 & 61,4 & 66,1 & 73,2 & 84,2 \\
\hline 3 & 44,4 & 63,9 & 65,5 & 74,0 & 80,2 & 88,8 & 102,0 \\
\hline 4 & 50,3 & 72,0 & 74,3 & 83,6 & 90,6 & 99,9 & 115,3 \\
\hline 5 & 52,5 & 75,4 & 77,7 & 87,4 & 94,7 & 104,4 & 120,5 \\
\hline 6 & 27,1 & 38,4 & 39,9 & 44,6 & 48,7 & 53,4 & 62,2 \\
\hline 7 & 15,6 & 22,7 & 23,4 & 26,0 & 28,6 & 31,2 & 36,4 \\
\hline 8 & 8,1 & 11,5 & 12,2 & 13,3 & 14,4 & 15,6 & 18,5 \\
\hline 9 & 2,3 & 3,4 & 3,4 & 3,8 & 4,1 & 4,5 & 5,3 \\
\hline
\end{tabular}




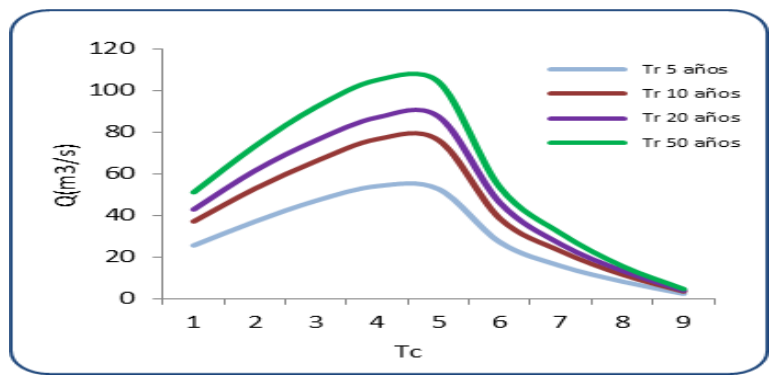

Gráfico 1: Caudal máximo subcuenca Huaycoloro

\subsection{Peligros Naturales - Inundación}

Las inundaciones han existido siempre desde la historia de la humanidad y obedecen a las características de la subcuenca respectiva, en el caso de la subcuenca Huaycoloro presentan una topografía accidentada con fuerte pendiente en la parte alta y media, y pendiente poco pronunciada en la parte baja, lo que origina que la quebrada Huaycoloro en épocas de avenida de enero a marzo originen según los períodos de retorno históricos que cada 10 o 15 años hay inundaciones, lo que se convierten en desastres, cuando el hombre debido a la comodidad de su vida cotidiana prefiere ubicarse cerca de los cursos de agua, así como también por la falta de necesidad de un terreno donde vivir, se ubica en las riberas de los ríos sin darse cuenta que es un peligro inminente.

La falta de agua y desagüe del centro poblado de Cajamarquilla obliga a la población y a las empresas que habitan en el lugar a descargar sus aguas residuales en la quebrada Huaycoloro, lo cual influye el aumento de caudal, lo que perjudica al centro poblado Huachipa que se encuentra en la confluencia con el Río Rímac.

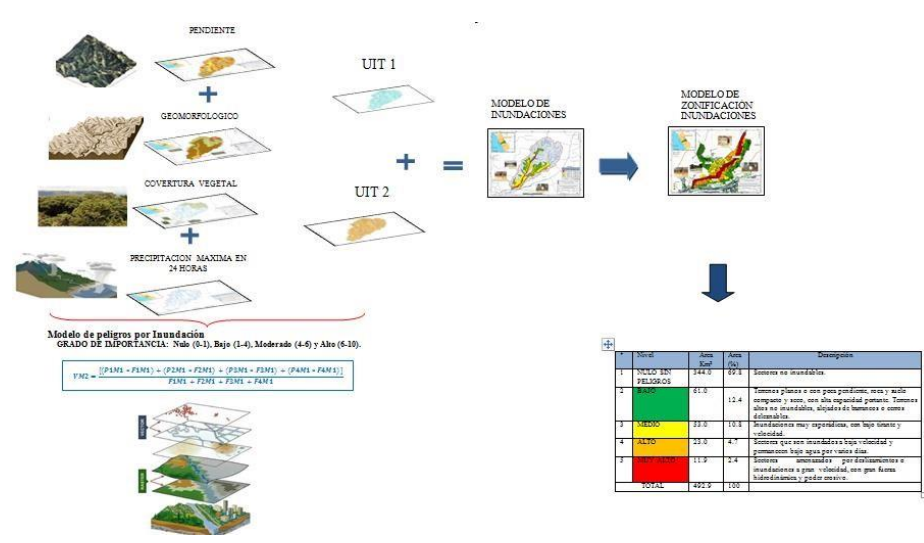

Gráfico 2: Ejecución del modelo de peligros por inundación. Fuente: Elaboración propia.
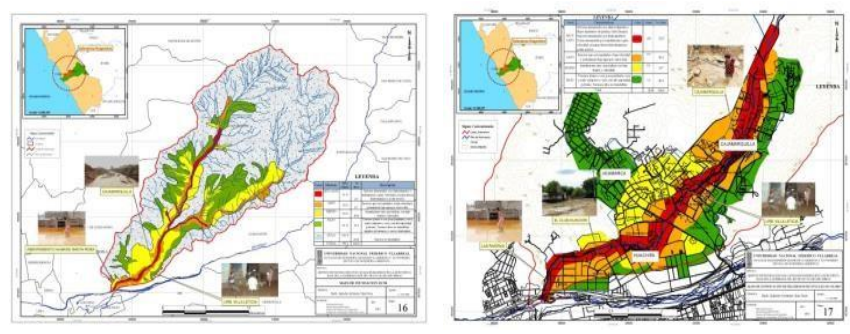

Mapa 1: Inundación a $10 \operatorname{Tr}$ y Zonificación de peligros. Fuente: Elaboración propia.

\subsection{Análisis de vulnerabilidad}

La vulnerabilidad, es la susceptibilidad de una unidad social (familias, comunidad, sociedad), estructuras físicas o actividad económica que la sustenta a sufrir daños por acción de un peligro o amenaza. La identificación de la vulnerabilidad sirve para analizar instalaciones o estructuras que tienen mayor riesgo y a qué nivel podrán ser afectadas. Una gran ayuda para esta tarea es contar con planos de la ciudad, de los sistemas de agua potable, desagüe, líneas de alimentación de energía eléctrica teléfono, vías de comunicación y el Mapa de peligros correspondiente.

Foto 1: Pozo de agua inutilizable, los pozos también sufrieron daños en casi su totalidad y originando los escases de agua para consumo.

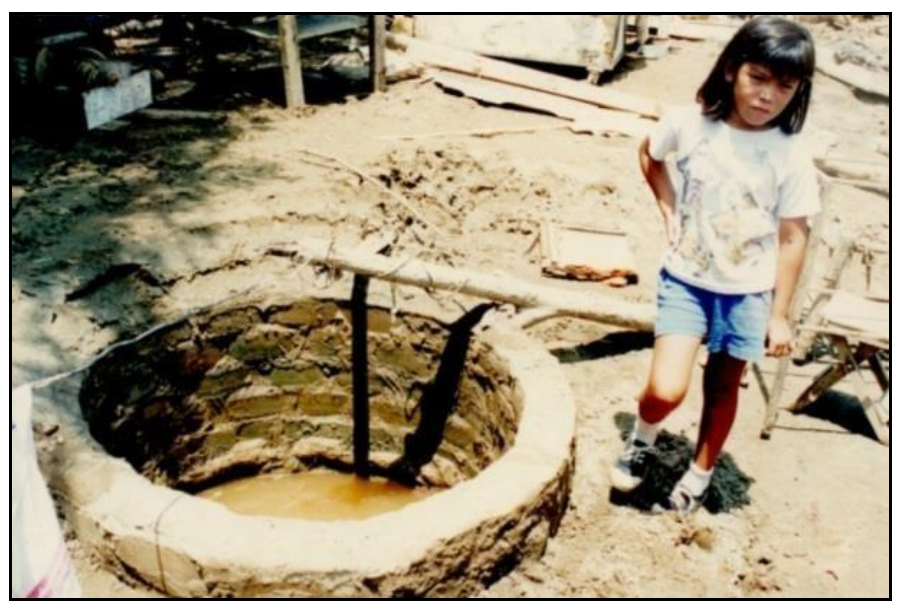

\subsection{Evaluación del riesgo}

Los daños y pérdidas producidas en la subcuenca Huaycoloro afectan a las viviendas, la vida humana, infraestructura de riego y vías públicas. 
Considerando análisis obtenidos en el análisis de vulnerabilidad se ha estimado la evaluación física y la valoración económica de los daños a un total de 200 viviendas que están expuestas.

Según el concejo nacional de tasaciones (CONATA), el valor unitario de vivienda de material de barro y ladrillo es de S/. 1867.2 y S/. 6821.2. Para lo cual se ha estimado un total de pérdidas de $\mathrm{S} /$.

$891,322.00$.

\section{Propuesta de conservación y protección de inundación.}

\subsection{Limpieza de y Mantenimiento del Cauce.}

- Objetivo: Mantener la subcuenca baja de la quebrada huaycoloro limpia, a fin de mitigar el impacto ante la activación de ocurrencia de inundación.

- Beneficiarios del Proyecto: Población asentada en zonas riesgos alto y muy alto como los asentamientos humanos. - Periodo de ejecución: 11 días.

- Costo: S/. 56, 612.75

- Institución Promotora: Municipalidad distrital de Lurigancho Chosica y Municipalidad de Lima. Tabla 3: Estimación económica de daños producidos a las viviendas.

\begin{tabular}{|c|c|r|c|c|}
\hline Vivienda & barro & $\begin{array}{c}\text { Valor } \\
\text { estimado en } \\
\text { S/. }\end{array}$ & ladrillo & $\begin{array}{c}\text { Valor } \\
\text { estimado en } \\
\text { S/. }\end{array}$ \\
\hline Santa rosa & 0 & S/. 0.00 & 4 & S/. 27,284.80 \\
\hline Riveras de Huachipa & 0 & S/. 0.00 & 1 & S/. 6,821.20 \\
\hline Villa Leticia & 10 & S/. 18,672.00 & 18 & S/. 122,781.60 \\
\hline Aviadores & 2 & S/. 3,734.40 & 10 & S/. 68,212.00 \\
\hline Pampa chiri & 18 & S/. 33,609.60 & 8 & S/. 54,569.60 \\
\hline Aras del huayco & 12 & S/. 22,406.40 & 10 & S/. 68,212.00 \\
\hline Residencial Cajamarquilla & 9 & S/. 16,804.80 & 9 & S/. 61,390.80 \\
\hline $\begin{array}{c}\text { Urb. Fundo Nievería 2 da } \\
\text { etapa }\end{array}$ & 4 & S/. 7,468.80 & 2 & S/. 13,642.40 \\
\hline Urb. La capitana & 15 & S/. 28,008.00 & 3 & S/. 20,463.60 \\
\hline Urb. El club & 0 & S/. 0.00 & 7 & S/. 47,748.40 \\
\hline Urb. Huachipa norte & 2 & S/. 3,734.40 & 3 & S/. 20,463.60 \\
\hline Urb. Huertos de Huchipa & 0 & S/. 0.00 & 0 & \\
\hline Urb. Brisas de Huchipa & 4 & S/. 7,468.80 & 1 & S/. 6,821.20 \\
\hline Asoc. Porcino saracoto & 19 & S/. 35,476.80 & 6 & S/. 40,927.20 \\
\hline La florida & 4 & S/. 7,468.80 & 8 & S/. 54,569.60 \\
\hline $\begin{array}{c}\text { Asoc. Pequeños criadores de } \\
\text { animales menores. }\end{array}$ & 24 & S/. 44,812.80 & 7 & S/. 47,748.40 \\
\hline total & 123.00 & 229665.60 & 97.00 & S/. 661,656.40 \\
\hline
\end{tabular}

Fuente: Elaboración propia.

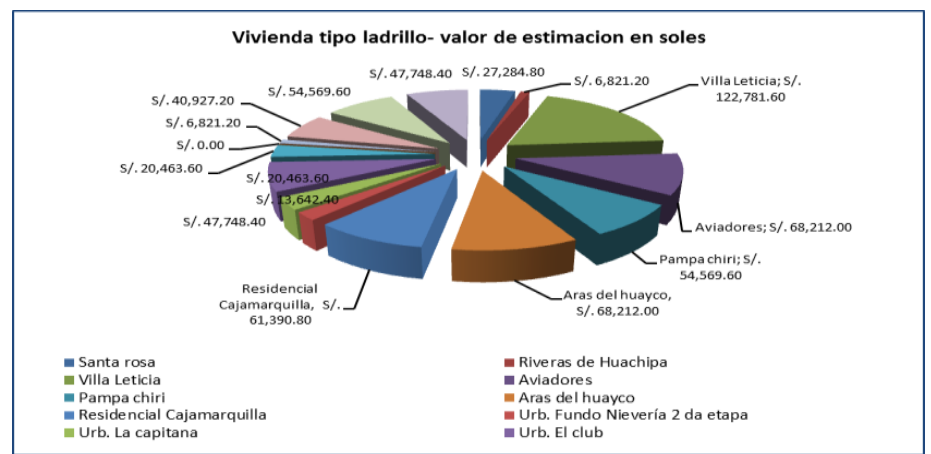

Gráfico 3: Vivienda tipo ladrillo. Fuente: Elaboración propia.

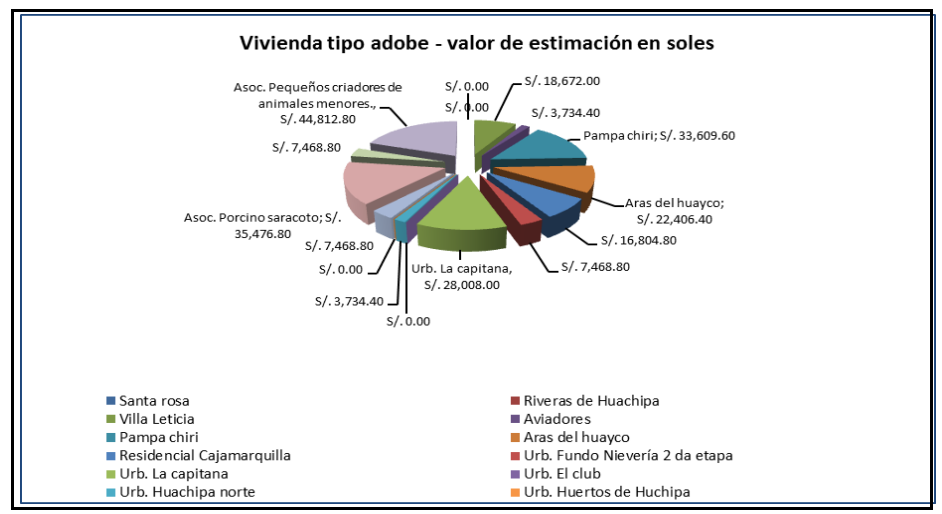

Gráfico 4: Vivienda tipo adobe. Fuente: Elaboración propia.

- Descripción: El proyecto propondrá los mecanismos necesarios, normativos, administrativos y de logística para que la municipalidad periódicamente realice limpieza del cauce de la quebrada baja del río Huaycoloro y retirando así el material inestable, comprometiendo a la población ubicada en la cercanía de las márgenes a participar del mantenimiento, considerando la vigilancia por parte de la misma población organizada, para evitar el arrojo de desechos sólidos y desmonte. Asimismo, disminuir la erosión lateral.

Tabla 4: Zonificación del riesgo de la Subcuenca Huaycoloro.

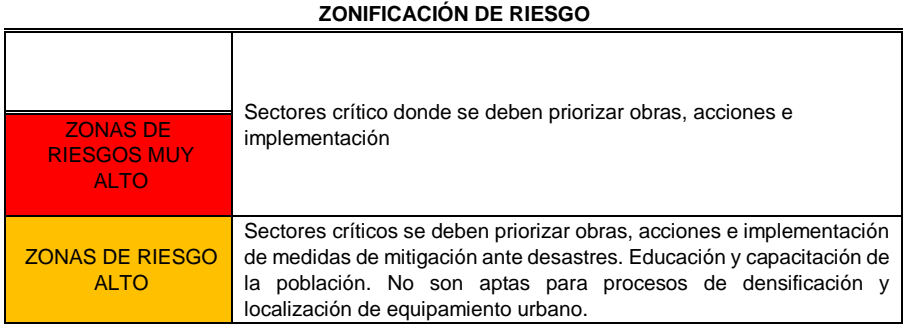




\begin{tabular}{|c|l|}
\hline $\begin{array}{c}\text { ZONAS DE RIESGO } \\
\text { MEDIO }\end{array}$ & $\begin{array}{l}\text { Suelos aptos para uso urbano. Es deseable implementación ante } \\
\text { desastres y educación y capacitación de la población en temas de } \\
\text { prevención. Pueden densificarse con algunas restricciones. Daños } \\
\text { considerables en viviendas en mal estado. }\end{array}$ \\
\hline $\begin{array}{c}\text { ZONAS DE RIESGO } \\
\text { BAJO }\end{array}$ & $\begin{array}{l}\text { Suelos aptos Para uso urbano de alta densidad y localización de } \\
\text { equipamientos urbanos de importancia, tales como hospitales grandes } \\
\text { centros educativos. Bombeos, cuarteles de policía, etc. } \\
\text { Daños menores en las edificaciones. }\end{array}$ \\
\hline
\end{tabular}

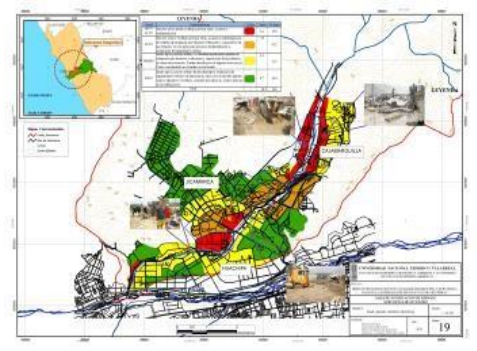

Mapa 2: Zonificación de riesgos Fuente: Elaboración propia.

\subsection{Muro de Gaviones Subcuenca Baja de la Quebrada del Río Huaycoloro.}

- Objetivo: Reducir los riesgos de inundación y desbordes en áreas críticas de las márgenes de la cuenca de la quebrada Huaycoloro, mediante la implementación de un programa de defensa ribereña y obras de encauzamiento.

- Beneficiarios del Proyecto: Población asentada en zonas riesgos alto y muy alto como los asentamientos humanos. - Periodo de ejecución: 26 días

- Costo: S/. 67, 786.40

- Descripción: El proyecto propondrá los mecanismos necesarios, administrativos y de logística para que la municipalidad realice la instalación de gaviones por su menor costo y durabilidad. A fin de reducir la velocidad y caudal de los flujos en su desplazamiento ante eventos extraordinarios. Se debe tener en cuenta el ancho estable del río, sin llegar a un estrechamiento excesivo, debe ser compatible con la tendencia natural del agua, considerando lo siguiente:

- Retención de sedimentos, sabiendo que, en un año o máximo en dos, las presas se colmatarán.

- Los muros deben tener la altura en función a la pendiente y el cálculo de flujos de lodo por año.

- La longitud del muro de gaviones está en función al ancho de la quebrada y de los niveles topográficos.

- La cimentación de las estructuras está en función de las características del basamento geológico de la zona.

\subsection{Muro de Llantas Subcuenca Baja de la Quebrada del Río Huaycoloro.}

Objetivo:

Disminuir la velocidad del escurrimiento y su poder erosivo.

Periodo de ejecución: 13 días

Costo: S/. 44,258.40

Descripción

La instalación de muros de llantas como medida para prevenir inundación y a la vez erosión ocasionadas por el poder fluvial favorece en esta propuesta por ser una medida ecológica debido al rehúso de llantas y por el menor costo de la obtención de los mismos. $Y$ por consiguiente se reforzarán con postes que tenga la función de darle la sostenibilidad al muro de gaviones. El proyecto propondrá los mecanismos necesarios, administrativos y de logística para que la municipalidad realice la instalación de muros de llantas.

\subsection{Forestación Subcuenca Baja de la Quebrada Huaycoloro.}

- Objetivo: Favorecer la retención e infiltración de agua y la recarga de acuíferos. Periodo de ejecución: 2 meses.

- Costo: S/. 17,563.00

- Descripción: La forestación desempeñaría un papel importante en la subcuenca baja de la quebrada del rio Huaycoloro debido a que cumple una función en el ciclo hidrológico por tanto es imprescindible como una medida natural de mitigación para inundaciones, asimismo su costo y mantenimiento es económico, asimismo mejorar la belleza paisajística de la zona en riesgo y así disminuirlos para evitar daños a la población.

- Mejora el comportamiento hidrológico de la cuenca, favoreciendo en la llanura de inundación.

- El almacenamiento de agua

- El retraso de avenidas

- La reducción de los daños por erosión de márgenes

- La retención de escorrentías y sedimentos

- La retención de nutrientes

Tabla 5: Especies Utilizadas para la Reforestación. Fuente: Elaboración propia.

\begin{tabular}{|l|l|c|c|c|}
\hline Variables & \multicolumn{3}{|l|}{ Especies utilizadas para la reforestación } \\
\cline { 2 - 5 } & $\begin{array}{c}\text { Tara } \\
\text { (Caesalpinia } \\
\text { Spinosa) }\end{array}$ & $\begin{array}{c}\text { Molle } \\
\text { (Schinus } \\
\text { molle) }\end{array}$ & $\begin{array}{c}\text { Yaravisco, } \\
\text { Leucaena } \\
\text { (leucaena } \\
\text { leucocephala) }\end{array}$ & $\begin{array}{c}\text { Sauce (Salix } \\
\text { chilensis) }\end{array}$ \\
\hline
\end{tabular}




\begin{tabular}{|c|c|c|c|c|}
\hline Agroforestería & $\begin{array}{l}\text { Cerco viv } \\
\text { o, barreras } \\
\text { vivas. }\end{array}$ & 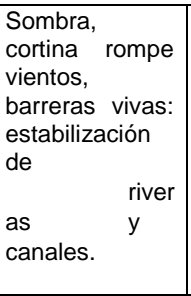 & $\begin{array}{l}\text { Cercos vivos, } \\
\text { barreras vivas, } \\
\text { estabilización } \\
\text { de cárcavas, } \\
\text { taludes, } \\
\text { prácticas de } \\
\text { conservación } \\
\text { de suelos. }\end{array}$ & $\begin{array}{l}\text { Conservación } \\
\text { del suelo } \\
\text { restauración } \\
\text { de zonas } \\
\text { ribereñas } \\
\text { erosionadas } \\
\text { Barrera } \\
\text { rompe } \\
\text { vientos; } \\
\text { Cerca vivo. }\end{array}$ \\
\hline Medicinal & $\begin{array}{l}\text { Amigdalitis, } \\
\text { cicatrizante, } \\
\text { estomatitis, } \\
\text { gripe, fiebre. }\end{array}$ & $\begin{array}{l}\begin{array}{l}\text { Antirreumático, } \\
\text { purgante, } \\
\text { cicatrizante, } \\
\text { depurativo de la } \\
\text { sang } \\
\text { re, riñón, vejiga. }\end{array}\end{array}$ & $\begin{array}{l}\text { Malestares } \\
\text { bronquiales }\end{array}$ & $\begin{array}{l}\text { Fiebres, } \\
\text { reumatismo } \\
\text { bronquitis. }\end{array}$ \\
\hline Plaguicidas & $\begin{array}{l}\text { Contra Piojos } \\
\text { insectos }\end{array}$ & polillas & $\begin{array}{l}\text { Repelente de } \\
\text { insectos }\end{array}$ & \\
\hline madera & & $\begin{array}{l}\text { Carpintería } \\
\text { y } \\
\text { ebanistería. }\end{array}$ & $\begin{array}{l}\text { Poder } \\
\text { calorífico }\end{array}$ & $\begin{array}{l}\text { Canastas, } \\
\text { muebles, } \\
\text { cajones y } \\
\text { construcción } \\
\text { rural. } \\
\end{array}$ \\
\hline Otros & $\begin{array}{l}\text { Caída del } \\
\text { cabello, } \\
\text { Color negro y } \\
\text { azul }\end{array}$ & $\begin{array}{l}\text { Perfumería, } \\
\text { cremas } \\
\text { dentales, } \\
\text { Amarillo }\end{array}$ & $\begin{array}{l}\text { Ornamental, } \\
\text { beta caroteno. }\end{array}$ & Ornamental. \\
\hline $\begin{array}{l}\text { Área total a } \\
\text { reforestar }\end{array}$ & 18 ha & 12 ha & 16 ha & $18 \mathrm{ha}$ \\
\hline $\begin{array}{l}\text { Costo total de } \\
\text { la plantación } x \\
\mathrm{Ha}\end{array}$ & 2000.00 & 1500.00 & 1250.00 & 1250.00 \\
\hline
\end{tabular}

\subsection{Educación Ambiental y Prevención de Desastres}

Objetivo: Establecer un sistema de respuestas apropiadas para enfrentar un evento de alto riesgo producido por la inundación estructurando un sistema de organización equipamiento y logística adecuada ante la emergencia.

Periodo de ejecución: Corto, Mediano y largo plazo.

Costo de Inversión: S/. 39, 600.00

Descripción: El proyecto propondrá los mecanismos necesarios, normativos, administrativos y de logística para que la municipalidad periódicamente deba realizar con la finalidad de educar a la población ante la ocurrencia de riesgos por inundación identificando así los peligros y las vulnerabilidades que estos provocan. Eventos potenciales de riesgos por inundación son: - Derrumbes de viviendas y saturación de los suelos.

- Ruptura de las tuberías de desagüe y obstrucción de la misma.

Incendios por humedecimientos de las líneas eléctricas enterradas.

Rupturas de caminos, carretas y puentes.

Daños al drenaje de la ciudad.

Daños a terceros, incremento de los costos de los alimentos y otros.
Según el volumen 7 de la evaluación del potencial hidroeléctrico nacional le asigna a la cuenca del Rímac una precipitación anual de $50 \mathrm{~mm}$.

El inventario de evaluación nacional de aguas superficiales 1982 determina para la quebrada de 165 $\mathrm{mm}$.

La estación Antioquia que se encuentra cuya altitud es similar a la altitud media de la Subcuenca tiene una precipitación media de $110 \mathrm{~mm}$; pudiéndose asignar de la precipitación anual de la cuenca está en el rango de 110 - $165 \mathrm{~mm}$ de lluvia anual.

\section{Conclusiones}

Se realizó el diagnostico físico, bilógico, ambiental, y la determinación del riesgo en el ámbito de estudio. En lo físico su área de la subcuenca Huaycoloro es de $492,9 \mathrm{~km}$, red de drenaje de orden 5 , un perímetro $112.7 \mathrm{~km}$ y de altitud $1678 \mathrm{msnm}$. Así también el diagnostico socioeconómico resalta que la pobreza de los 38436 habitantes el $60 \%$ se encuentra en pobreza extrema y el resto regular; los asentamientos humanos expuestos a los niveles de peligros por la exposición de residuos sólidos y material de desmonte que impiden el pase del cauce.

Se determinó los caudales para la simulación hidráulica de la subcuenca Huaycoloro con los periodos de retorno de 5 de $53 \mathrm{~m} 3 /$ hasta $121 \mathrm{~m} 3 / \mathrm{s}$ para 100 años periodos de retorno; que corresponden las precipitaciones máximas en 24 horas para esos mismos periodos de retorno asimismo se determinaron lo hidrogramas de caudales. Contribuyendo en el análisis de zonas con mayor peligro y vulnerabilidad debido a las características hidráulicas y sociales de la población determinando para ello las zonas de muy alto y alto riesgo. Asimismo, se estimó la pérdida económica en viviendas es de S/. 891,322.00; perdidas económicas en la población S/. 220,320.0; infraestructura de vías y canales es de S/. 76,175.10 y S/. 102,994.50 respectivamente.

Se plantean 5 propuestas de mitigación, las cuales constituyen 4 de protección (Limpieza y mantenimiento del cauce, muro de gaviones, muro de llantas, Educación ambiental y prevención de desastres) con un presupuesto de S/. 56,612, S/. 67 , S/.786., S/. 44,258, S/. 39, 600 respectivamente y 1 de conservación (forestación) con presupuesto de S/. $17,563.00$ y un tiempo de sostenibilidad para 5 años.

\section{Agradecimientos}

\section{Discusiones}


Dr. Walter Gómez Lora: Hoy, director del instituto de investigación de la FIGAE-UNF, docente de la cátedra de Hidrología y Manejo de cuencas.

Dr. Noé Zamora Talaverano, asesor de la Universidad Federico Villarreal y docente de la cátedra de Sistema de Información Geográfico.

A los estudiantes del CEIGA, Centro de Investigación y Gestión del Agua; por su tiempo y valiosas opiniones en al aporte de la etapa de campo.

\section{Referencias}

[1] J. Gómez Lora, Tesis Maestría con el tema Gestión de Inundación del Río Rímac (2000), Lima-Perú

[2] INDECl, "Manual para la Estimación de Riesgos Ante Inundaciones Fluviales", 2011.
[3] IPROGA, (El Instituto de Promoción para la Gestión del Agua), muestra la "Metodología para la Elaboración de Planes Maestros de Cuencas", PROMACHS, 1를 Edición, Perú, 1996.

[4] Vent Chow, David R. Maidment \& Larry W. Mays, una herramienta para el análisis de los tiempos de retorno y coeficiente de escorrentía. "Hidrología Aplicada". Segunda Edición. D'vinni Editorial: Ltada, BogotáColombia, 1994.

[5] INGEMMET. "Geología de los Cuadrángulos de Lima, Lurín y Chosica; hojas 25 j, 24 j, 25 i, 24 i",1992.

E-mail: ginad_quincho@hotmail.com 